Neurogenesis and angiogenesis inhibitors

The monoclonal antibody (mAb) Avastin (bevicuzumab) is an angiogenesis inhibitor targeting vascular endothelium growth factor (VEGF) approved for the

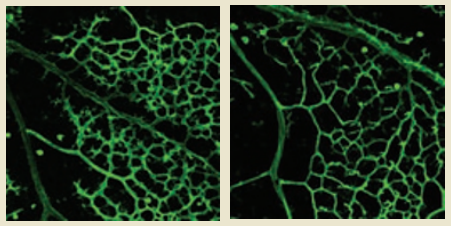
treatment of colon and lung

cancers. Because some tumors do not respond or become resistant to Avastin therapy, researchers at Genentech (S. San Francisco, CA, USA) set out to establish whether a mAb targeting the neuropilin system - axon guidance molecules that bind to a particular isoform of VEGF found on tumors and endothelial cells key to the process of vascular remodeling-might boost the efficacy of VEGF mAbs. Using mAbs against neuropilin receptors, they observed inhibition of endothelial cell migration and vessel sprouting both in vitro and in vivo, similar to VEGF mAbs. The neuropilin receptor mAbs, however, did not affect VEGF-mediated changes in endothelial cell permeability, proliferation or receptor autophorphorylation, suggesting that neuropilins act independently of, or in parallel with, VEGF. In a xenograft tumor model, the researchers show an additive effect on tumor-growth inhibition when the neuropilin $\mathrm{mAbs}$ are applied in combination with VEGF mAbs. Neuropilins appear to render endothelial cells more dependent on VEGF, a finding that indicates the system may represent a promising target for anticancer therapeutics. (Cancer Cel/ 11, 53-67, 2007)

\section{Reawakening the gatekeepers}

Maintenance of tumorigenesis is thought to require persistent oncogene activation. Three studies now demonstrate that cancers in mouse models also require continued inactivation of tumor suppressors for their maintenance. In the first, Ventura et al. use a Cre-loxP-based strategy to restore $p 53$ expression at the endogenous loci in primary, autochthonous lymphomas and sarcomas that develop in $p 53$ null mice; some of these mice were irradiated after birth to decrease tumor latency. Upon tumor detection by whole-body imaging, reinstatement of $p 53$ expression by unsequestering cytoplasm-localized Cre resulted in significant tumor regression. Reactivation of $p 53$ caused apoptosis and cell cycle arrest with senescence in lymphomas and sarcomas, respectively. In the second report, Xue et al. use RNA interference to conditionally regulate endogenous p53 expression in an athymic nude mouse model of liver carcinoma seeded from embryonic liver progenitor cells transduced with oncogenic ras (HrasV12) and a regulable p53 short hairpin RNA construct. They show that reactivation of endogenous p53 for 4 days in p53-deficient tumors caused complete regression by inducing tumor-cell senescence and a concomitant tumor-targeted innate immune response. In the last study, Martins et al. demonstrate that restoration of one wild-type $p 53$ locus in oncogenic $E \mu$-myc lymphomas with a $p 53$ null background triggers tumor apoptosis and significantly increases survival. In contrast to the first two studies, p53 reinstatement in the presence of oncogenic Myc selected for the emergence of p53-resistant tumors through inactivation of $\mathrm{p} 19^{A R F}$ or $\mathrm{p} 53$. Although all three papers suggest that drugs or gene therapies that reinstate wild-type tumor suppressors might prove effective cancer therapeutics, long-term tumor regression might not be possible in certain genetic backgrounds. (Nature 445, 661-665, 2007; Nature 445, 656-660, 2007; Cell 445, 1323-1334, 2006)

JWT

Research Highlights written by Kathy Aschheim, Laura DeFrancesco,

Peter Hare, Ania Levinson \& Jan-Willem Theunissen.

\section{Multivalency key to dendrimers}

Dendrimers — highly branched monodisperse polymers_-are being explored as delivery vehicles for enhancing the therapeutic index of cancer drugs. One type of dendrimer, 5- poly(amidoamine) (PAMAM) molecules conjugated to multiple folic acid molecules, shows enhanced targeting efficiency for cancer cells bearing folic acid receptors both in vitro and in vivo. Although the presumption has been that this improved delivery is due to multivalent interactions, no direct evidence of this has been gathered. Hong et al. now construct a series of dendrimer-based scaffolds with 2-14 folate molecules, testing them against a cell line overexpressing folate receptor. Surface plasmon resonance shows that the dissociation constants of PAMAM dendrimers are enhanced by up to five orders of magnitude over those of free folic acid. Using fluorescently labeled dendrimers to follow cellular uptake, the authors demonstrate that PAMAM molecules with multiple folate moieties bind to the cell surface rather than undergoing internalization, suggesting that the key to improved targeting efficiency is multivalency, not increased endocytosis. (Chem. Biol. 14, 107-115, 2007)

$A L$

\section{Targeted arthritis therapy?}

A drawback of existing therapies for rheumatoid arthritis is that they nonspecifically suppress or modulate pro-inflammatory cytokines, thereby increasing patient susceptibility to infection. But investigations into cadherin-11, a homotypic cellular adhesion protein involved in the development of the synovial lining of joints, suggest a more specific therapeutic approach. Using cadherin-11 null mice, as well as a mouse strain that spontaneously develops autoimmune arthritis, Lee et al. demonstrate the essential role of cadherin-11 in development of both the synovium and the organized synovial tissue response that accounts for much of the cartilage damage associated with arthritis. Studies involving the mouse $\mathrm{K} / \mathrm{BxN}$ serum transfer model of autoimmune inflammatory arthritis indicate that administration of either a cadherin-11-Fc fusion protein or a cadherin-11 monoclonal antibody can attenuate the onset of acute inflammation, the latter also ameliorating established arthritis. These findings offer promise for ongoing efforts to direct arthritis therapies more specifically to sites associated with disease inflammatory processes. (Science, $315,1006-1010)$

$\mathrm{PH}$

\section{Predicting genomic enhancers}

The system of gene regulation inscribed on the genome in the form of histone modifications remains largely obscure. Ren and colleagues sought to decipher this 'histone code' by analyzing chromatin modifications in 44 loci of the human genome, chosen because they are relatively well studied, which totaled $30 \mathrm{Mb}$ in length. Chromatin immunoprecipitation-chip experiments on HeLa cells show that promoters and enhancers have distinct signatures: promoters are trimethylated on Lys4 of histone H3, whereas enhancers are monomethylated on this same residue. The authors then use these results to develop a predictive algorithm and identify $\sim 400$ potential enhancers in the $30-\mathrm{Mb}$ region, several of which are validated with reporter assays. Thus far, large-scale identification of enhancers has proved challenging. The approach represents a new strategy for functional annotation of the human genome and possibly the genomes of other organisms. (Nat. Genet., published online 4 February 2007, doi:10.1038/ng1966)

$K A$ 